\title{
Correction to: Antifibrotics in liver disease: are we getting closer to clinical use?
}

\author{
Meena B. Bansal ${ }^{1}$ Naichaya Chamroonkul ${ }^{1,2}$
}

Published online: 30 January 2019

(c) Asian Pacific Association for the Study of the Liver 2019

\section{Correction to: Hepatology International https://doi.org/10.1007/s12072-018-9897-3}

In the original publication, the following text "Printed with permission from (OMount Sinai Health System" had been missed to include from Figs. 1 to 4 captions. The correct figure legends are given below.

Fig. 1 Hepatic myofibroblasts are originated from heterogenous types of fibrogenic cell. Activated hepatic stellate cells are considered to be a source of liver fibrogenic cells. Portal fibroblasts have also been demonstrated as a source of myofibroblast especially in cholestatic liver disease. Furthermore, reported minor proportion of liver fibrogenic cells includes bone marrow-derived cells and epithelial-mesenchymal transition (EMT). Printed with permission from CMount Sinai Health System

Fig. 2 Following liver damage, hepatic stellate cells transform from quiescent vitamin A-rich cells into fibrogenic myofibroblast. The major phenotypic changes after activation include proliferation, contractility, fibrogenesis, matrix degradation, chemotaxis and WBC chemoattraction. During resolution of liver injury, population of activated stellate cells may be reduced by transdifferentiating their phenotype back to a quiescent formed, cellular senescence and/or apoptosis. Printed with permission from @Mount Sinai Health System

The original article can be found online at https://doi.org/10.1007/ s12072-018-9897-3.

\section{Meena B. Bansal}

meena.bansal@mssm.edu

1 Division of Liver Diseases, Icahn School of Medicine at Mount Sinai, 1425 Madison Avenue, Box 1123, New York, NY 10029, USA

2 Division of Gastroenterology and Hepatology, Department of Internal Medicine, Faculty of Medicine, Prince of Songkla University, Hatyai, Songkhla 90110, Thailand
Fig. 3 Multiple signals and pathways involve in processes of HSC activation. Key fibrogenic and proliferative mediators contribute to fibrogenesis, include tissue growth factor-b (TGF-b1), platelet-derived growth factor (PDGF), vascular endothelial growth factor (VEGF), and connective tissue growth factor (CTGF). Hedgehog (Hh) ligand and its receptor smoothened homolog (SMO). G proteincoupled receptors including cannabinoid receptor $1 / 2$ receptors (CB1, CB2), 5-hydroxytryptamine receptors (5HTs), proteinase-activated receptor 2 (PAR2), $\mathrm{C}-\mathrm{C}$ chemokine receptors (CCRs), succinate dehydrogenase-G protein-coupled receptor 91 (GPR91), type 1 angiotensin II receptor (AT1R) can affect HSC activation. Innate immune signalling induced by Toll-like receptors (TLRs), lipopolysaccharide, and damage-associated molecular patterns (DAMPs) has been implicated in stellate cell activation. Cytokines and chemokines mediate crosstalk between hepatic stellate cells and others non-parenchymal liver cells such as kupffer cell are also critical features in liver fibrogenesis. Endoplasmic reticulum (ER) stress and oxidative stress are features of chronic liver disease that activates HSCs. Alteration of nuclear receptors expression also involves in stellate cell activation both in positive and negative ways. Epigenetic signals including microRNAs (mi RNAs), DNA methylation and histone modification control both activation and inactivation of HSCs. FXR, farnesoid X receptor; LXR, liver $X$ receptor; NR4A1, nuclear receptor subfamily 4 group A member 1; PPARs, peroxisome proliferator-activated receptors; VDR, vitamin D3 receptor; Rev-erb, nuclear receptor subfamily 1 , group $\mathrm{D}$, member 1 . Printed with permission from $\odot$ Mount Sinai Health System

Fig. 4 Mechanisms by which antifibrotic therapies may lead to fibrosis regression. [1] Reduction of HSC activation by reducing hepatic inflammatory response including removed injurious stimuli, introduced antioxidant, introduced hepatic protection agents and applied immune modulation. [2] Inhibition of the most potent of the profibrogenic pathways, for example, preventing expression of 
profibrogenic mediators, blocking hepatic stellate cell proliferation and reducing contraction of hepatic stellate cells. [3] The resolution of fibrosis can be promoted by enhancing the apoptosis of activated hepatic stellate cells and by increasing the degradation of the extracellular matrix or preventing its cross-linking with antagonists to LOXL2. CCR2/ CCR5, C-C chemokine receptor 2 and 5, FXR, farnesoid $\mathrm{X}$ receptor; PPAR, peroxisome proliferator-activated receptor; CB1, cannabinoid receptor type 1; ACE-I, angiotensin converting enzyme inhibitors; TGFb, transforming growth factor b; siRNA of HSP47, small interfering RNA of heat shock protein 47; TIMP, tissue inhibitor of metalloproteinase; LOXL2, lysyl oxidase 2. Printed with permission from CMount Sinai Health System

Publisher's Note Springer Nature remains neutral with regard to jurisdictional claims in published maps and institutional affiliations. 